\title{
A study on the effects of the alcoholic extract of the aerial parts of Echinophora platyloba on the activity of pituitary-gonadal axis in hypercholesterolemic rats
}

\author{
Sokhandani Mansour ${ }^{1}$, Zarei Ali $^{2^{*}}$, Changizi-Ashtiyani Saeed ${ }^{3}$ \\ ${ }^{1}$ Nursig Department, Shiraz University of Medical Sciences, Shiraz, Iran. ${ }^{2}$ Department of Physiology, Abadeh Branch, Islamic Azad University, Abadeh, \\ Iran. ${ }^{3}$ Department of Physiology, Arak University of Medical Sciences, Arak, Iran .
}

\begin{tabular}{|c|c|}
\hline ARTICLE INFO & ABSTRACT \\
\hline Article history: & \multirow{9}{*}{$\begin{array}{l}\text { The present experimental study was carried out to examine the effects of the alcoholic extract of the aerial parts } \\
\text { of Echinophora platyloba on prolactin (PRL) levels and the activity of pituitary-gonadal axis in animal model. } \\
\text { Forthy male wistar rats were divided into five groups: control group with normal diet, hypercholesterolemic } \\
\text { group with fat diet, and three experimental groups which received Echinophora platyloba alcoholic extract as } \\
\text { gavage at the minimum dose of } 100 \text {, the average dose of } 200 \text {, and the maximum dose of } 400 \mathrm{mg} / \mathrm{kgbw} \text { over a } \\
\text { period of } 21 \text { days. At the end of the period, to assess the levels of PRL, cholesterol, testosterone, luteinizing } \\
\text { hormone (LH) and follicle stimulating hormone (FSH), blood samples were taken and data were analyzed. The } \\
\text { levels of testosterone and cholesterol in hypercholesterolemic group compared to the control, showed a } \\
\text { significant decrease and increase, respectively }(P \leq 0.05) \text {. Echinophora platyloba extract caused significant } \\
\text { increases in testosterone, PRL and a significant decrease in cholesterol levels }(P \leq 0.05) \text {, however, it had no } \\
\text { significant effect on the levels of LH and FSH. The results of this study showed that the consumption of } \\
\text { alcoholic extract of Echinophora platyloba is effective in reducing serum cholesterol, increasing testosterone } \\
\text { production and enhancing sexual performance. }\end{array}$} \\
\hline Received on: 12/04/2016 & \\
\hline Revised on: 07/05/2016 & \\
\hline Accepted on: 15/06/2016 & \\
\hline Available online: $28 / 07 / 2016$ & \\
\hline Key words: & \\
\hline Echinophora platyloba, & \\
\hline Prolactin, Pituitary gland, & \\
\hline Testosterone, Rats. & \\
\hline
\end{tabular}

\section{INTRODUCTION}

The impact of obesity on fertility has long been known. Aristotle (384-322 BC), when investigating the causes of infertility, emphasized on its role. The famous Iranian physician, Rhazes (854-932A.D) in his book Al-Hawi, recommended diet and exercise to obese people for their problem of infertility (Tsoucalas et al., 2014; Ramezani et al., 2013). Today, about 10 to 15 percent of couples worldwide are infertile. In developing countries one out of four couples are infertile and in Iran there are about 3 million infertile couples (Bokaie et al., 2012). Presently, obesity has become epidemic and is growing quickly in all developing countries and a lot of researches have been

\footnotetext{
* Corresponding Author

Young Researchers and Elite Club, Abadeh Branch, Islamic Azad University, Abadeh, Iran.

Tel+989171502855, E-mail: zareiali47@yahoo.com
}

carried out to determine the relationship between hormones, cholesterol and reproductive activities. Prolactin is introduced as a potential factor for diabetes and hyperlipidemia (Klenov, Jungheim, 2014; Ramezani Tehrani, 2012). In obese men, the levels of testosterone, sex hormone-binding globulin (SHBG) and sometimes the plasma free testosterone are reported to be lower than in normal people (Binder et al., 2015). Male infertility has many causes and in most cases, the medication therapy is carried out, without necessarily identifying the cause of infertility (Jungwirth et al., 2012). Nowadays, however, due to the adverse effects and the side effects of chemical drugs, traditional medicine, especially the use of herbal medicine has become so trendy (Ekor, 2014). In recent years many studies have been carried out to learn about the effects of different plants on the fertility of laboratory animals and the results have been so promising (Hatami and Estakhr, 2013). In this study, too, the effects of Echinophora platyloba on reproductive activity was examined. 
Echinophora is among the flora of Iran, which includes species like E. cinerea, E. platyloba, E. orientalis, E. sibthorpina. This plant belongs to the family of Apiaceae with the local names of tigh tooragh, koshandar, khosharizeh (Entezari et al., 2014; Avijgan et al., 2010).

Among the main constituents of this plant one can refer to myrcene, ocimene, monoterpenes, saponins, flavonoids and alkaloids (Delaram, Haeri, 2011). People add its aerial parts to cheese and yogurt as fragrant. The extract of this plant has antioxidant, anti-fungal and anti-bacterial and anti-spasmodic effects; it is also used for the treatment of menstrual disorders (Delaram Haeri, 2011; Aqababa et al., 2016). Flavonoids are among the main components of the plant, which have direct effects on the pituitary gland leading to increased secretion of luteinizing hormone ( $\mathrm{LH})$, followed by increased secretion of progesterone in women (Shobeiri et al., 2012).

So far no study has been carried out to examine the effect of the plant on the male reproduction system. However, as it has been effective on the levels of $\mathrm{LH}$ and steroid hormones in women, it seems that the extract of this plant may be effective on the pituitary-gonadal axis, as well. The main objective of this experimental study was to investigate the effects of the alcoholic extract of the aerial parts Echinophora platyloba on prolactin (PRL) levels and the activity of pituitary-gonadal axis (HPG) in Wistar adult male rats.

\section{MATERIALS AND METHODS}

\section{Animals}

This was an experimental study on male Wistar rats. During the entire process, the ethical codes for using laboratory animals in accordance with "principles of laboratory animal care" (NIH publication no. 85-23, revised 1985) were fully observed. The animals were taken from Shiraz branch of Razi vaccine and serum research institute and were studied at Abade PNU (Fars/ Iran).The animals were housed under standard laboratory conditions and 12 hours light / dark cycles at the temperature of 22- $26^{\circ} \mathrm{C}$ and were randomly divided into 5 groups of $8(n=8)$ as follows: The control group which did not receive any drug or solvent during the experiment and were treated with normal diet; the hypercholesterolemic group that daily received $0.2 \mathrm{ml}$ of solvent (saline) as gavage during the experimental period; the experimental groups with hypercholesterolemic rats receiving daily the alcoholic extract of Echinophora platyloba by gavage at a dose of 100 (minimum dose); the hypercholesterolemic rats which received daily the alcoholic extract of Echinophora platyloba as gavage at a dose of 200 (average dose); and the hypercholesterolemia rats which daily received the alcoholic extract of Echinophora platyloba at a dose of $400 \mathrm{mg} / \mathrm{kg}$ bw (maximum dose) (Heidarian et al,2014).

The experimental period was 21 days during which the prescribed extracts and solvent were gavaged at 9 am .All groups including the control group were treated with high-cholesterol food. At the end of the period, by a mild anesthesia with ether, blood samples were taken from the heart, and after centrifugation at 3,000 rpm, the serums were separated and sent to the lab in order to measure their plasma biochemical factors.

\section{The protocol for the preparation of a $2 \%$ cholesterol diet}

To prepare a high-fat meal, cholic acid 5\% (Merck, 3671), cholesterol 2\% (Merck, 3672), and olive oil 5\% were used, respectively, in such a way that after heating 5 grams of olive oil up to $70^{\circ} \mathrm{C}, 2$ grams of cholesterol and 0.5 gram of colic acid would be dissolved in it.

The resulting solution was poured on 92.5 grams of dry food and then they were mixed. The resulting mixture covered the entire surface of the plates as a white gel. To prevent deterioration, the food was kept at $4^{\circ} \mathrm{C}$ in the fridge for only two days (Hatami and Estakhr, 2013; Khosravizad et al., 2016).

\section{Extraction protocol}

First the plant was compared with the herbarium code of 004-046-091 and confirmed by the botanical department of Abade Payame Noor University (PNU). For the preparation of the alcoholic extract, first in spring Echinophora-platyloba aerial parts were collected from Bavanat district of Fasa/Iran. The impurities were removed and the remaining were washed and dried in shade. Then 700 grams of the plant was ground and mixed with ethanol $90 \%$ at a ratio of 1 to 5 . After stirring for 24 hours, the resulting extract was filtered.

The remainder was again mixed with ethyl alcohol $70 \%$ and was placed on the stirring device for another 24 hours; afterwards, it was filtered and added to the first extract. Then the extract was placed in the vacuum distillation unit at the temperature of $60^{\circ} \mathrm{C}$ until the residual volume was one-fifth of the initial one. In this case, the extract tank was separated from the machine and the remainder was cooled for three times and each time was decanted with $50 \mathrm{cc}$ of chloroform; then the volume obtained was poured in Petri and dried in the oven at $50^{\circ} \mathrm{C}$. Finally, by adding normal saline to the obtained extract (about 11 $\mathrm{g}$ for $100 \mathrm{~g}$ of the crushed plant) different required concentrations were made in milligrams per kg .(Zarei et al., 2015; Zarei, Vaezi, 2015).

\section{The method of measuring the biochemical parameters}

Serum cholesterol levels were determined by using the kit from Darman Kav Co. (Iran) by means of colorimetry. To assess the levels of PRL, cholesterol, testosterone, LH and follicle stimulating hormone (FSH) the radioimmunoassay method (RIA) was used, together with a Pars Azmoon kit and RIA 1000 machine made in America (Zarei et al., 2014).

\section{Statistical analysis}

The means obtained (Mean \pm SEM) were statistically evaluated by using one way ANOVAs and Tukey and Duncan Tests. All statistical analysis was performed using SPSS software, version $23(P \leq 0.05)$. 
Table 1: The results (Mean \pm SD) of the effects of Echinophora-platyloba on plasma biochemical factors in different groups.

\begin{tabular}{|c|c|c|c|c|c|}
\hline parameters & Control & Hypercholesterolemic & $\begin{array}{l}\text { Echinophora platyloba } \\
\text { extract at a dose of } 100 \mathrm{mg} / \\
\text { kg (minimum dose) }\end{array}$ & $\begin{array}{c}\text { Echinophora platyloba } \\
\text { extract at a dose of } \\
200 \mathrm{mg} / \mathrm{kg} \text { (average } \\
\text { dose) }\end{array}$ & $\begin{array}{c}\text { Echinophora platyloba } \\
\text { extract at a dose of } 400 \\
\text { mg / kg } \\
\text { (maximum dose) }\end{array}$ \\
\hline (FSH) (MIU/ml) & $4.06 \pm 0.22$ & $4.10 \pm 0.16$ & $3.95 \pm 0.14$ & $4.36 \pm 0.18$ & $3.90 \pm 0.22$ \\
\hline$(\mathbf{L H})(\mathrm{MIU} / \mathrm{ml})$ & $7.75 \pm 0.14$ & $8.06 \pm 0.25$ & $37.38 \pm 0.24$ & $8.03 \pm 0.18$ & $8.38 \pm 0.30 \beta$ \\
\hline Testosterone (ng/ml) & $6.23 \pm 0.09$ & $5.30 \pm 0.18^{*}$ & $6.08 \pm 0.21$ & $6.26 \pm 0.24 \dagger$ & $7.48 \pm 0.28 \beta \dagger €$ \\
\hline Prolactin (MIU/ml) & $11.06 \pm 0.18$ & $11.18 \pm 0.33$ & $11.20 \pm 0.31$ & $12.03 \pm 0.36$ & $12.30 \pm 0.33$ \\
\hline Cholesterol (mg/dl) & $63.56 \pm 3.97$ & $75.14 \pm 2.31$ & $53.11 \pm 5.04 \dagger \alpha$ & $70.21 \pm 3.11$ & $51.52 \pm 3.55 \dagger \beta$ \\
\hline
\end{tabular}

*: significant changes in the control group compared to the hypercholesterolemic group.

$\dagger$ : significant changes in the experimental group compared to the control group.

$\alpha$ : significant changes between the experimental groups ( the average and the minimum).

$\beta$ : significant changes between the experimental groups (the maximum and the average).

$€$ : significant changes between the experimental groups (minimum and maximum).

\section{RESULTS}

A summary of the findings is shown in table 1-4. The level of cholesterol in the control group showed a significant increase compared to the hypercholesterolemic group. Its level in all experimental groups receiving the extract showed a significant decrease compared to the control group. In addition, cholesterol levels in experimental groups receiving the minimum dose (100 $\mathrm{mg} / \mathrm{kg} \mathrm{bw}$ ) and maximum dose (400 $\mathrm{mg} / \mathrm{kg} \mathrm{bw})$ of the extract showed a significant decrease in comparison to the rats receiving the average dose of the extract $(200 \mathrm{mg} / \mathrm{kg} \mathrm{bw}),(\mathrm{P} \leq 0.050)$, (Table 1).

The changes in FSH levels in all three experimental groups receiving the extract were not significant, either $(\mathrm{P} \leq 0.05)$. Also, in none of the groups receiving the extract, LH levels showed significant changes in comparison to the control group; but LH level in the group receiving the maximum dose compared to the group receiving the minimum dose showed a significant increase $(\mathrm{P} \leq 0.050)$. The testosterone levels in the experimental group receiving the highest dose of the extract showed a significant increase in comparison to those in the rats receiving the minimum and average doses of it $(\mathrm{P} \leq 0.05)$. Prolactin levels in the group receiving the maximum dose of the extract showed a significant increase compared to the control group (Table 1).

\section{DISCUSSION}

The results of this study showed that testosterone levels in the hypercholesterolemic group decreased compared to the control group and also, the testosterone and PRL levels in the group receiving the maximum dose of Echinophora platyloba extract (400 mg/kg bw) increased compared to the control group. $\mathrm{LH}$ and FSH levels in the experimental groups receiving the extract did not show significant changes compared to control and hypercholesterolemic groups. The level of cholesterol in the control group increased compared to the hypercholesterolemic group but it decreased in the experimental groups receiving the extract (100 and $400 \mathrm{mg} / \mathrm{kg} \mathrm{bw}$ ) compared to the control group. Given that the control group received a diet containing cholesterol, the increased level of cholesterol in this group seems to be reasonable. But, as stated before, in the hypercholesterolemic experimental groups receiving the extract the cholesterol levels decreased. Studies done by Khosravi zad et al. (2015) and Aghabab et al. (2015) on the hypolipidemic property of the extract also showed that the extract of this plant reduced blood lipid profiles (Aqababa et al., 2016; Khosravizad,2016).

The results of this study are consistent with the above mentioned studies. The importance of the phytochemical effects of medicinal herbs on weight loss and obesity has been known for many years. Saponins, flavonoids, alkaloids, coumarin and terpenes are some of these compounds which are also present in Echinophora platyloba. In addition to its anti-tumor properties, saponin has cholesterol-lowering effects (Singh S, Kanwar, 2013). Alkaloids also act as cholesterol synthesis inhibitors (ChangiziAshtiyani, 2013). So, it seems reasonable if cholesterol level decreases by receiving Echinophora platyloba extract.

Hyperlipidemia and obesity, particularly upper body fat, cause many hormonal changes in the body including: increased serum androgen levels in women and its decreased levels in men, increased cortisol and insulin and decreased serum levels of growth hormone (Binder,2015). As was observed in this study, in the hypercholesterolemic group testosterone levels decreased that is consistent with the results of the above studies. Moreover, the previous studies on Echinophora platyloba showed that this extract could help reduce weight (Aqababa et al., 2016). Although, there are contradictory results in some studies concerning the blood fat level and the level of sex hormones, most studies confirm that there is a reasonable relation between testosterone levels and the measured fat (Binder et al., 2015).

Studies done by Delaram et al. (2010) on the effect of Echinophora platyloba on symptoms of premenstrual syndrome, sex hormone changes and gonadotropin hormones showed that the extract of the plant increased the secretion of $\mathrm{LH}$, followed by an increase in the secretion of progesterone, that probably occurred due to the presence of flavonoids which ,in its turn, by acting on the pituitary gland caused an increase in LH and subsequently an increase in progesterone levels (Delaram, Haeri, 2011, Delaram, Sadeghiyan,2010). In the present study, which was conducted on male rats, in the groups receiving the extract, the increase in the level of LH was not significant compared to that in the control group, but the amount of testosterone in the group receiving the extract showed a significant increase. 
The fact that no significant increase in the levels of FSH and LH were seen in experimental groups receiving the extract might be due to the increased levels of testosterone and PRL since the increased testosterone level exerted a negative feedback on LH; moreover, PRL can inhibit the FSH secretion. It's a fact which is clearly recognized even in breast-feeding women who have high levels of PRL. Prolactin prevents the follicle growth by inhibiting $\mathrm{FSH}$ and eventually ovarian follicle development during lactation (Allahtavakoli et al., 2015).

The results of the present study also revealed that this plant extract increased PRL level. Ramezani and colleagues (2013) also showed that obesity was associated with lower levels of sex hormone-binding globulin (SHBG).

They found that in patients with metabolic syndrome the levels of androgens were high and SHBG level was low. The researchers also showed that there was an inverse relationship between serum PRL and waist, while Cook reported the increased PRL secretion in obese patients (Cook, 2005). In this study, the PRL level in hypercholesterolemic group showed no significant changes in comparison to the control group. So, there are conflicting studies about the relationship between PRL levels and obesity (Ramezani Tehrani etal., 2012).

An inverse correlation between leptin and serum testosterone levels has been observed (Luukkaa et al ,1998). Leptin is a peptide that is secreted from adipose tissue which acts through reducing food intake and increasing metabolism as signals to maintain energy balance in the body and its secretion is inhibited by testosterone. On the other hand, leptin's effect on its receptor in Leydig cells of the testes can regulate testosterone production. Obesity, testosterone and leptin in men seem to be closely related and this relationship may be exerted by one of the following mechanisms:

A- Obesity may reduce testosterone levels and thus eliminate its inhibitory effect on leptin and subsequently increase leptin serum.

B- Obesity may be associated with increased leptin, and its inhibitory effect on testosterone levels may reduce testosterone.

C- Low levels of testosterone and high levels of leptin may occur after obesity independent of each other.

D- Low testosterone production may lead to obesity and subsequent increase in leptin. In men, high levels of leptin and lower levels of serum testosterone and SHBG are associated with body weight; moreover, high concentration of leptin and low concentration of testosterone independent of body mass index (BMI) are associated with each other. The inverse association between leptin and serum testosterone possibly has a role in reducing serum testosterone levels in obese individuals (Binder et al. 2015, Ceschia et al., 2007). As stated, the plant extract reduces blood fat levels. Also, by reducing leptin and removing its inhibitory effect on testosterone, it increases the level of testosterone.

The study by Aqababa et al. (2015) on the effect of Echinophora platyloba showed that the extract of this plant in maximum dose had a hepatoprotective effect, as well as reducing liver enzymes and elevating the levels of total protein and albumin in hypercholesterolemic rats. The results also showed that testosterone levels also increased in the group receiving the extract of this plant which was probably due to the effect of this plant on SHBG which helped the transmission of the steroid hormones (Aqababa et al., 2016).

The positive effect of the medicinal plants with warm nature used in traditional medicine, such as fennel, dill, cumin, nettle, and fenugreek, on the secretion of PRL and milk has been confirmed. Phenolic compounds are among the main ingredients prescribed even in oral drops to increase milk production. Due to the warm nature of the plant and having abundant phenolic compounds that can increase the secretion of PRL, possibly this plant has the potential to increase milk production (Avijgan et al., 2010; Khazaei et al., 2011).

Studies done by Khosravi Zad and colleagues (2015) showed that the extracts of the plant increased the secretion of FSH. This is likely done after the increase of the secretion of thyrotropin releasing hormone (TRH) from the hypothalamus (Khosravizad et al., 2016). Prolactin secretion is controlled by a PRL inhibitory factor with hypothalamic origin. The hypothalamus also produces a PRL -releasing hormone (PRLH), which stimulates the secretion of PRL (Allahtavakoli et al., 2015).

\section{CONCLUSION}

Regarding the results of this study and others on the extract of Echinophora platyloba, it appears that the use of this plant extract is effective on reducing blood lipids, increasing the secretion of testosterone and strengthening sexual activity because of its antioxidants and lipid-lowering compounds, such as alkaloids and flavonoids. The effects of this herb on improving sexual activity, in addition to the possible effects of the extract on the secretion of sex hormones, are possibly exerted through reducing lipid profiles, and also a weight loss mechanism.

\section{ACKNOWLEDGEMENTS}

This article is taken from a research project approved by Shiraz University of Medical Sciences, and Abade Hazrat Zahra Nursing School with the approval no. 8337-93-01-93. We appreciate the research deputy of Shiraz University of Medical Sciences and Hazrat Zahra Nursing School who helped us in conducting this study.

\section{COMPETING INTERESTS}

The authors declared no potential conflicts of interest. 


\section{REFERENCES}

Allahtavakoli M, Honari N, Pourabolli I, Kazemi Arababadi M, Ghafarian H. Vitex agnus castus extract improves learning and memory and increases the transcription of estrogen receptor $\alpha$ in hippocampus of ovariectomized rats. Basic Clin Neurosci. 2015 ;6:185-92.

Aqababa H, Golkary H, Zarei A, Changizi Ashtiyani S. Effect of aerial parts of Echinophora platyloba.1 on liver and kidney function tests in obese hypercholesterolaemia rats. J Shahid Sadoughi Univ Med Sci 2016; 23: 943-56.

Avijgan M, Mahboubi M, Darabi M, Saadat M, Sarikhani S, Kassaiyan N. Overview on Echinophoraplatyloba, a synergistic antifungal agent candidate. Journal of Yeast and Fungal Research, 2010; 1: 88-94.

Binder NK, Sheedy JR, Hannan NJ, Gardner DK. Male obesity is associated with changed spermatozoa cox4il mRNA level and altered seminal vesicle fluid composition in a mouse model. Mol Hum Reprod. 2015;21:424-34.

Bokaie M, Farajkhoda T, Enjezab B, Heidari P, Karimi Zarchi M, .Barriers of child adoption in infertile couples: Iranian's views Iran J Reprod Med. 2012; 10: 429-434.

Ceschia M, Gutzwillerb f, Mochc H, Eichholzerb M, Nicole M. Probst-henscha. epidemiology and pathophysiology of obesity as a cause of cancer. swiss med wkly. 2007;137:50-56.

Changizi-Ashtiyani S, Zarei A, Taheri S, RezaeiA. A comparative study of hypolipidemic activities of the extracts of Melissa officinalis and Berberis vulgaris in rats. Journal Medicinal Plants. 2013;12: 38-47.

Cook DM Long-term management of prolactinomas--use of long-acting dopamine agonists. Rev Endocr Metab Disord. 2005;6:15-21.

Delaram M, Haeri A. The effect of Echinophora platyloba on the pre-menstural syndrome. research in medicine. 2011;34:219-224.

Delaram M, Sadeghiyan Z. The effect of Echinophora platyloba extract on primary of dysmenorrhea. Arak University of Medical Sciences Journal. 2010; 13:61-67.

Ekor M.The growing use of herbal medicines: issues relating to adverse reactions and challenges in monitoring safety. Front Pharmacol. 2014;4:177.

Entezari M, Dabaghian FH, Hashemi M. The comparison of antimutagenicity and anticancer activities of Echinophora patyloba DC on acute promyelocytic leukemia cancer cells. J Cancer Res Ther. 2014;10:1004-7.

Hatami L, Estakhr J. The effects of hydroalcoholic extract of matricaria recutita on the hormonal pituitary-testis axis and testis tissue changes of mature male rats. Journal of Fasa University of Medical Sciences . $2013 ; 3: 57-62$.

Heidarian E, Saffari J, Jafari-Dehkordi E. Hepatoprotective action of Echinophoraplatyloba DC leaves against acute toxicity of acetaminophen in rats. J Diet Suppl. 2014;11:53-63.

Jungwirth A, Diemer T, Dohle GR,. Giwercman A. European Association of Urology guidelines on Male Infertility: the 2012 update. Eur Urol $2012 ; 62: 324-32$.
Khazaei M, Montaseri A, Khazaei MR, Khanahmadi M. Study of foeniculum vulgare effect on folliculogenesis in female mice. Int $\mathbf{J}$ Fertil Steril. 2011; 5: 122-127.

Khosravizad M, Zarei A, Chobineh MA, Karimi F. Study the effect of air Echinophora-platylobaon the pituitary-thyroid axis and the lipid profile in hypercholesterolemic rats. J Gorgan Uni Med Sci. 2016 (in Press).

Klenov VE, Jungheim ES. Obesity and reproductive function: a review of the evidence. Curr Opin Obstet Gynecol. 2014;26:455-60.

Luukkaa V, Pesonen U, Huhtaniemi I, Lehtonen A, Tilvis R. Inverse correlation between serum testosterone and leptin in men. J Clin Endocrinol Metab. 1998 ;83:3243-6.

Ramezani M, Changizi-Ashtiyani S, Shamsi M, Taheri S. The opinion and views of Rhaze,s, Avicenna,s and Jorjani,s on fertility and infertility . Complementary Medicine Journal. 2013;3:504-505.

Ramezani Tehrani F, Zadeh Vakili A, Hashemi S, Amuzegar A, Azizi F. Associations between hormonal profiles of urban Iranian women of reproductive age: A community based study of four provinces. Iranian Journal of Endocrinology and Metabolism. 2012,14: 32-38.

Singh S, Kanwar SS. Therapeutic effect of herbal medicines on obesity: herbal pancreatic lipase inhibitors. Journal of Medicinal Plants. 2013; 2(4):53 - 65

Shobeiri F, Zeraati F, Mansouri Z, Araghchian M, Nazari M . The comparative effect of herbal extract of vitagnus and mefenamic acid on primary dysmenorrhea. Zahedan J Res Med Sci. 2012; 14: 30 - 33.

Tsoucalas G, Karamanou M, Laios K, Androutsos G. The pioneer physician euryphon and his method for determination of the female infertility in ancient Greece. J Obstet Gynaecol. 2014;34:369.

Zarei A, Changizi-Ashtiyani S, Vaezi GH. A study on the effects of the hydroalcholic extract of the aerial parts of Alhagicamelorum on prolactin and pituitary-gonadal activity in rats with hypercholesterolemia .Archivio Italiano di Urologia e Andrologia 2014; 86, 3:188-193.

Zarei A, Vaezi Gh, Malekirad AA, Abdollahi M. Hypoglycemic and hypolipidemic activities of salvia hydrangea in streptozotocin-induced diabetes in rats. Iran J Basic Med Sci 2015; 18: 417-422.

Zarei A, Vaezi Gh, Malekirad AA, Abdollahi M. A comparison of the effects of ethanol extract of salvia hydrangea on hepatic and renal functions of streptozotocin-induced diabetic rats. Avicenna Journal of Phytomedicine. 2015,5: 138-1448.

\section{How to cite this article:}

Sokhandani Mansour, Zarei Ali, Changizi-Ashtiyani Saeed., A study on the effects of the alcoholic extract of the aerial parts of Echinophora platyloba on the activity of pituitary-gonadal axis in hypercholesterolemic rats. J App Pharm Sci, 2016; 6 (07): 115-119. 